Case report: cobblestone

\title{
Ejaculatory pain associated with a pelvic arteriovenous malformation
}

\author{
R M Angunawela, D F C Shepherd, M J Hayward, A H De Silva
}

\section{Introduction}

Genital discomfort and pain associated with ejaculation are common presenting symptoms at a genitourinary clinic. We present a patient with such symptoms arising from a congenital pelvic arteriovenous malformation.

\section{Case report}

A 22 year old man presented with severe suprapubic pain on ejaculation. The pain lasted several hours after sexual intercourse and at times occurred spontaneously. Penile erection was accompanied by throbbing pelvic pain. Defaecation was occasionally painful. There was no haematuria, haematospermia, or dysuria.

Physical examination revealed a lower abdominal bruit. A pulsatile mass was felt at the left lateral border of the prostate gland. There were no external signs of a vascular abnormality. Limb development was symmetrical. Sigmoidoscopy was normal. The prostate gland and seminal vesicles appeared normal on transrectal ultrasonography. Routine screening excluded sexually transmitted infections.

Magnetic resonance imaging (MRI) showed an arteriovenous malformation (AVM) behind the bladder and between the left seminal vesical and upper prostate gland. Angiography confirmed a complex high flow AVM fed mainly from branches of the left internal iliac artery (see fig 1).

He was treated elsewhere and, after two courses of embolisation, was asymptomatic.

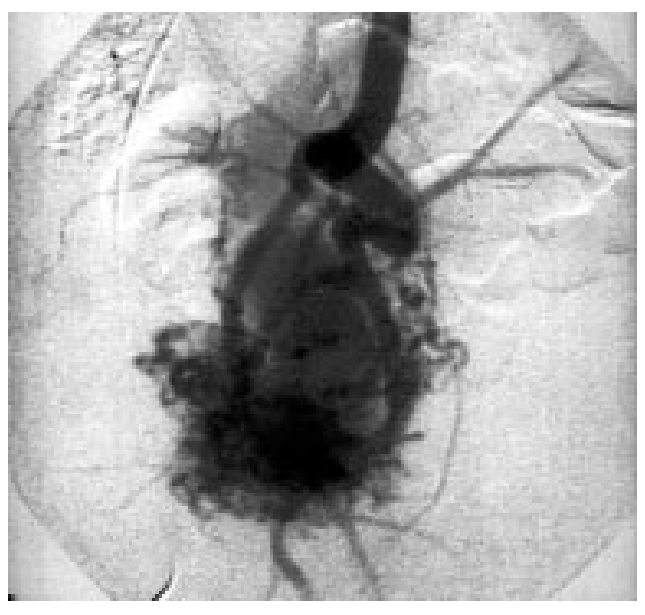

Figure 1 Pelvic angiogram showing arteriovenous malformation arising from left internal iliac artery.

\section{Discussion}

Congenital AVMs are uncommon and are usually localised to the extremities or head and neck. Congenital pelvic AVMs are particularly rare in males. ${ }^{1}$ AVMs result from developmental arrest of embryonic vascular channels. They may exist as an isolated mass or may be incorporated in pelvic soft tissue-for example, bladder, bowel. They enlarge in size and consequently become symptomatic in response to the hormonal stimulation of puberty or pregnancy. $^{2}$

Suprapubic discomfort and perineal and erectile pain are common presenting features. Genitourinary symptoms include urgency, hesitancy, frequency, dysuria, haematuria, and impotence. Scrotal oedema and ipsilateral limb hypertrophy may occur. Most pelvic AVMs, however, are silent and are often found serendipitously. Physical signs include a lower abdominal bruit and a pulsatile tender mass palpated on abdominal or rectal examination. Haemorrhoids and perineal varicosities may be seen externally. Haemodynamic sequelae such as cardiac failure are unusual.

Transrectal colour Doppler ultrasonography is suitable for initial non-invasive evaluation. Computed tomography and MRI followed by selective arteriography are required to define the extent and architecture of the vascular anomaly.

Asymptomatic patients with non-expanding lesions should be monitored at 6 monthly intervals. Surgical excision or ligation has largely been superseded by percutaneous embolisation which cures or affords symptomatic relief in $40 \%$ of patients. This yield is increased with repeated embolisation. Preoperative embolisation may be used as an adjunct to surgery to reduce the extent of the lesion and render it amenable to complete surgical excision. The optimal method of treatment is best decided jointly by the vascular surgeons and radiologist.

\footnotetext{
The authors thank Angela Fowler and Sue Stocker for secretarial assistance.

Contributors: DFCS conducted radiological investigation of the patient; all authors were collectively involved in making the diagnosis, collecting, reference search, and writing the manuscript.

Funding: Nil

Conflict of interest: Nil.

1 Calligaro KD, Sedlacek TV, Savarese RP, et al. Congenital pelvic arteriovenous malformation: long term follow up in two cases and a review of the literature. $f$ Vasc Surg 1992;16:100-8.

2 Rosen RJ, Riles TS. Arteriovenous malformation. In: Stradness DE Jr, Van Breda A, eds. Vascular diseases. Surgical and interventional therapy. New York: Churchill Livingstone, 1994:1121-37.
} 